\title{
Optical studies on Tb3+: Dy3+ singly and doubly doped borosilicate glasses for white light and solid state lighting applications
}

\begin{abstract}
Host (H), Singly and co-doped Dy3+/Tb3+ ions borosilicate glasses (S1-S7) were fabricated by a traditional melt-quenching-annealing method to investigate their luminescence properties as well as their suitability for the development of SSL (solid-state lighting) and W-LEDs (white light-emitting diodes). The X-ray diffraction profile indicates the amorphous structure nature of the prepared glasses. The J-O (Judd-Ofelt) intensity parameters $\Omega \mathrm{t}(\mathrm{t}=2,4,6)$ are evaluated for both singly Dy3+, Tb3 + doped glasses according to their absorption spectrum. The $\Omega 2$ parameter of Dy3+ ion has higher value which indicates a higher degree of Dy3+ covalency and the neighbour ligands while $\mathrm{Tb} 3+$ ion in a lower degree of covalency where its $\Omega 2$ has low value. Also the radiative parameters for both singly doped ions (S1 and S2) are computed like probability of transition (AT), branching ratio $(\beta r)$ and radiative lifetime $(\tau r)$ for different bands. Further, both singly doped glasses reflects good properties for lasing application where the 4F9/2 manifold of S1 (0.5 mol\% Dy3+) has radiative lifetime ( $\tau$ r) value of $1376 \mu \mathrm{s}$ and higher value of (AT:447.02 s-1) in addition to branching ration of $(\beta \mathrm{r}: 61.5 \%)$ for the $(4 \mathrm{~F} 9 / 2 \rightarrow 9 \mathrm{H} 13 / 2)$ transition $(576 \mathrm{~nm})$. At the same time, $\mathrm{S} 2(0.5 \mathrm{~mol} \% \mathrm{~Tb} 3+)$ glass show a higher lifetime ( $\tau \mathrm{r}: 3753)$ for 5D4 level and (AT: $92.94 \mathrm{~s}-1)$ while the branching ratio for $5 \mathrm{D} 4 \rightarrow 7 \mathrm{~F} 5$ band at $546 \mathrm{~nm}$ wavelength is ( $\beta \mathrm{r}: 34.9 \%)$. The radiative emission properties were investigated according to the $\mathrm{Tb} 3+$ concentration increments. The mutual energy transfer (ET) occurrence between both ions ( $\mathrm{Dy} 3+, \mathrm{Tb} 3+)$ is demonstrated through the varying of their emission intensities. Furthermore, The chromaticity $(\mathrm{x}, \mathrm{y})$ color coordinates were evaluated according to the emission spectra of aforementioned glasses under excitation of $368 \mathrm{~nm}$ and $348 \mathrm{~nm}$ were found to be located in the white light color region of the CIE chromaticity color diagram. Also, from color-coordinated the color correlated temperature (CCT) is computed were found to be at high values (>4000). Thus, the fabricated glasses are suggested as a useful luminescence material for solid-state lightening as well as cool white light applications.
\end{abstract}

Keyword: Borosilicate glass; Optical absorption; Radiative properties; Judd-Ofelt analysis; Luminescence; Energy transfer; W-LEDs 